\title{
One-shot Assistance Estimation from Expert Demonstrations for a Shared Control Wheelchair System
}

\author{
Ayse Kucukyilmaz* and Yiannis Demiris*
}

\begin{abstract}
An emerging research problem in the field of assistive robotics is the design of methodologies that allow robots to provide human-like assistance to the users. Especially within the rehabilitation domain, a grand challenge is to program a robot to mimic the operation of an occupational therapist, intervening with the user when necessary so as to improve the therapeutic power of the assistive robotic system. We propose a method to estimate assistance policies from expert demonstrations to present human-like intervention during navigation in a powered wheelchair setup. For this purpose, we constructed a setting, where a human offers assistance to the user over a haptic shared control system. The robot learns from human assistance demonstrations while the user is actively driving the wheelchair in an unconstrained environment. We train a Gaussian process regression model to learn assistance commands given past and current actions of the user and the state of the environment. The results indicate that the model can estimate human assistance after only a single demonstration, i.e. in one-shot, so that the robot can help the user by selecting the appropriate assistance in a human-like fashion.
\end{abstract}

\section{INTRODUCTION}

A 2012 report of the European Commission predicts a potential shortfall of around 1 million healthcare workers by 2020 , indicating a $15 \%$ reduction in the total work force when compared to the numbers in 2010 [1]. This significant shortage of nursing professionals implies an immediate need for developing assistive technologies capable of aiding the increasing elderly and disabled population. This deficiency, along with the advancements in the development of adaptive personal robotic systems, have motivated many researchers to turn their attention towards assistive robotic systems, which are tailored to aid humans with physical and/or cognitive impairments. The use of such robots has the potential to reduce the burden involved in caring for the increasing population of elderly and/or disabled people in frequently under-resourced clinical settings, such as rehabilitation and residential care centers [2].

Robotics technology is expected to have a positive impact on the quality of life of potential users with motor impairments by automating the rehabilitation process, offering precise monitoring tools, and providing adaptive assistance via modeling the behavior of the user. Up to now, many physical rehabilitation robots have been built to enhance the recovery of gait and upper extremity functions of patients

This work is supported by TUBITAK 2219 International Postdoctoral Grant, Turkey and EU grant "PAL": Personal Assistant for Healthy Lifestyle, grant H2020-PHC-643783.

*The authors are with the Personal Robotics Laboratory, Department of Electrical and Electronic Engineering, Imperial College London, Exhibition Road, SW7 2BT, London, UK \{a.kucukyilmaz,y.demiris\}@imperial.ac.uk

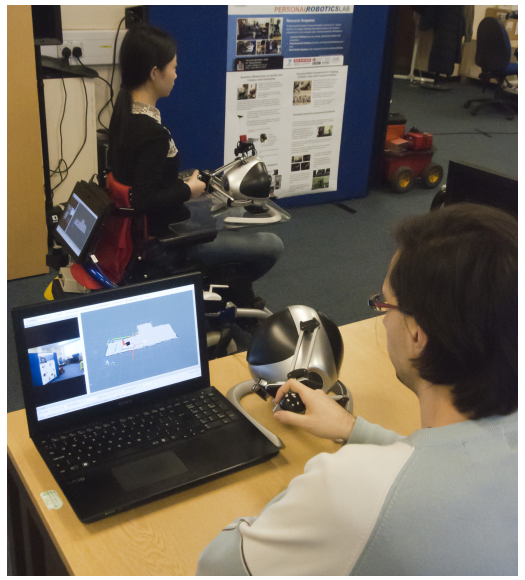

Fig. 1: Learning-Assistance-by-Demonstration Scenario: A remote assistant provides assistance to the user while she drives the wheelchair. The robotic system, in turn, learns human-like assistance through the observations of the environment and the assistant commands.

with motor deficiencies. Conventional approaches involve restricting the user's motion on a desired trajectory [3] or performing corrective actions when users deviate from the specified trajectory [4], [5], [6] during repetitive movement exercises. However, such solutions typically constrain the users motion, limiting the current effectiveness for therapeutic purposes [7], [8].

Since each individual has different needs and requirements for coping with specific motor impairments, methodologies that are able to provide personalized assistance to the users are needed. The fundamental point underlying such methodologies is that assistive systems should not unconditionally assist the user at all times, but attempt to infer the current needs of the user, and act accordingly [9]. We suggest that an assistive robot would be most effective if it can emulate the operation of a human assistant, e.g. an occupational therapist. Even though different studies have investigated humanhuman interaction to learn from the behavioral mechanisms utilized by humans (e.g. [10], [11], [12], [13]), there is no fundamental approach that allows researchers to develop a model for the robot so that it can act like a human assistant.

For this purpose, this study proposes a triadic learningby-demonstration (LbD) methodology, in which an assistive robot (i.e. a powered wheelchair) models its assistance function by observing the demonstrations given by a remote human assistant as shown in Fig. 1. LbD has been applied to human-robot interaction for over a decade to enable robots to imitate tasks performed by a human. In this methodology, 
the human acts as the "teacher", and physically demonstrates the task for the robot to generalize from [14], [15], [16]. Up to now, LbD has mostly focused on dyadic human-robot interaction scenarios, where the human is involved in the action only during demonstration. However, the wheelchair training scenario considered in this paper is inherently different than typical LbD scenarios. In our setup, the teacher is not the user of the wheelchair, but a remote human assistant, who interacts with the robotic system when needed. Additionally, since the user is continuously interacting with the wheelchair during the demonstration phase, it is not possible to formulate this problem as a typical LbD problem. The triadic nature of the problem requires the robotic system to be dynamic and responsive to different signals acquired from both the teacher (i.e. the assistant) and the user, while keeping track of what is going on within the environment. Up to our knowledge, such complex scenarios, where the demonstrations are given by a human assistant while the user and the robot pursues interaction, has not yet been considered except for a recent work by Soh and Demiris [9].

This paper is organized as follows: Section II presents related work on intelligent wheelchair systems. Section III details the hardware specifications of the haptics-enabled wheelchair system used as a test-bed, followed by Section IV, which explains the proposed methodology. Section V presents the experimental protocol followed in the user trials, and elaborates on data collection. Section VI presents a discussion of the results, and finally, Section VII provides conclusions and draws directions for future research.

\section{BACKGROUND}

Various studies have been conducted since mid-1990's as efforts to make powered wheelchair systems intelligent. Even though earlier studies typically implemented collision avoidance mechanisms for safeguarding (e.g. [17], [18], [19], [20]), in early 2000s, researchers have started to focus their efforts on estimating user goals and intentions using machine learning techniques to provide personalized assistance in powered wheelchair setups. Boy et al. proposed a mechanism that modulates the intelligent wheelchair's trajectory corrections based on the user's capabilities by defining specific control mechanisms tailored to specific disabilities [21]. Carlson and Demiris defined certain actions in a particular environment, and dynamically predicted the most probable actions that shall be taken in near future to correct the orientation of the wheelchair [22], [23]. Demeester et al. used Bayesian decision theory to estimate the certainty of users in a navigation task [24].

A challenging aspect of the intelligent wheelchair research domain is the fact that the users of the wheelchairs are often not able to provide correct signals to the wheelchair. This is especially apparent for toddlers and when wheelchair users suffer from extreme cognitive or physical difficulties. In such cases, since the users are not capable of performing all actions by themselves, it is not possible to construct a consistent assistive model based solely on user behaviors. That is why almost all existing wheelchair systems implement restrictive guidance schemes for strict trajectory-following tasks. A recent study by Soh and Demiris proposed that a robotic wheelchair can learn from the demonstrations of another person, who can present the user human guidance during navigation by taking over the control of the wheelchair [9], [25]. Assuming the coherence of human guidance, the robotic wheelchair modeled the user's actions and learned how and when to assist the user. In the current study, we take this idea one step further by learning the assistance policies during triadic interaction, where the assistant does not take over the control of the task, but shares control with the user at all times. This way, the user is given more autonomy in situations where (s)he comes up with navigation plans different from those of the assistant.

\section{SySTEM OVERVIEW}

This section describes the haptics-enabled wheelchair platform, through which the remote human assistant presents assistance to the user within a shared control framework.

\section{A. Robotic Platform}

In the experiments, we used the ARTY (Assistive Robotic Transport for Youngsters) intelligent wheelchair platform, developed at Imperial College London Personal Robotics Laboratory. ARTY is built by modifying a pediatric powered wheelchair to equip three Hokuyo URG-04LX laser scanners, five bump sensors, and one Phidgets IMU. The software system is developed using the Robot Operating System (ROS), and each sensor is managed by a separate ROS node. ARTY has two on-board computational units, which are responsible for processing and integrating multisensory information, as well as higher-level tasks, such as path planning and obstacle avoidance. More information on the operation of ARTY and its hardware specifications can be found in [26] and [27].

\section{B. Haptic Shared Control}

In our setup, the agents control the movement of the wheelchair with haptic joysticks. As a result, they get physically coupled, so that both are able to feel the forces exerted by one another as if they are holding the same joystick.

Each agent interacts with a Novint Technologies Falcon haptic controller with up to $9 \mathrm{~N}$ continuous force feedback capability and 400 dpi position resolution. The user's haptic controller is attached to ARTY and connected to one of the computational units on it, whereas the assistant's controller is connected to a remote PC that communicates with ARTY over the network. The wheelchair is controlled by moving the haptic controller on a horizontal plane, where the forward/backward movements are used for controlling the speed and leftward/rightward movements are used for rotation.

The operations of the user and the assistant are combined to calculate a joint command that is sent to the wheelchair in order to move it. The shared control scheme is based on the haptic negotiation model as described in [28]. ${ }^{1}$ Fig. 2 depicts

\footnotetext{
${ }^{1}$ ROS package that implements haptic negotiation for two Novint Falcon devices is accessible through the software section of the Personal Robotics Laboratory page: http://imperial.ac.uk/PersonalRobotics
} 
the modified haptic negotiation model that enables shared control on the wheelchair. The user and the assistant interact with the wheelchair by moving the haptic controllers, the positions of which are mapped to haptic interaction points $\mathrm{HIP}_{1}$ and $\mathrm{HIP}_{2}$ in the virtual world, where $\mathrm{HIP}_{1}$ denotes the user's haptic interaction point and $\mathrm{HIP}_{2}$ denotes that of the assistant. The operations of the agents are merged by introducing a negotiated interface point (NIP) that is directly connected to the HIPs through virtual spring-damper systems with zero rest lengths. In order to get the wheelchair to stop whenever the haptic joysticks are idle, NIP is also attracted towards the origin through a spring-damper system. Finally, the position of NIP is converted to a velocity command that is directly fed back to the wheelchair.

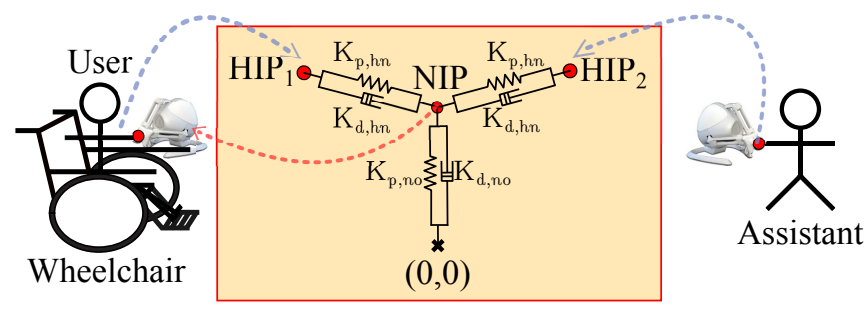

Fig. 2: Modified haptic negotiation model for controlling the movement of ARTY. $x_{i}$ and $\dot{x}_{i}\left(i \in\left\{\mathrm{HIP}_{1}, \mathrm{HIP}_{2}, \mathrm{NIP}\right\}\right)$ respectively denote the positions and velocities of $\mathrm{HIP}_{1}, \mathrm{HIP}_{2}$, and NIP; $K_{p}$ and $K_{d}$ values stand respectively for proportional and derivative gains: $K_{p, h n}=K_{p, n o}=15.0 \mathrm{~kg} / \mathrm{s}^{2}$, $K_{d, h n}=0.25 \mathrm{~kg} / \mathrm{s}$, and $K_{d, n o}=0.2 \mathrm{~kg} / \mathrm{s}$.

This model allows the user and the assistant to interact bidirectionally, being aware of one another's actions through the forces they feel via haptic devices. However, note that the humans feel each other's movements only indirectly through the NIP. In practice, the agents are fed back with very small forces as long as their movements agree. On the other hand, any disagreement between agents exposes itself immediately in terms of opposing forces fed back through the devices. This information is particularly important for the assistant so that (s)he can offer more effective guidance by getting aware of the actions of the user.

\section{APPROACH}

In general terms, we are interested in making an inference about the relationship between observation inputs, which consist of the environmental information and the user's control commands, and real-valued targets, which can replace the assistance signals as presented by a human. Regression presents a solution to this problem by learning the conditional distribution of the targets given the inputs [29]. In this study, we utilized a Gaussian process (GP) method for regression. GP is a powerful non-parametric Bayesian technique that describes data distributions over functions. It works very well with time-series, automatically handles temporal dependencies between input signals, provides probabilistic predictions, and presents a well-formulated Bayesian approach to model selection.
In the scope of this study, we used a squared exponential covariance function, which can model short and medium term irregularities in input space [29]. An initial lengthscale hyperparameter corresponding to 10 seconds is used to initialize the model. We believe this is a reasonable assumption since the length-scale of the process denotes the minimum distance you need to move along a particular axis in input space for the function values to be automatically uncorrelated. The final length-scale hyperparameter was optimized using maximum likelihood estimation during model selection. Due to the high-dimensional input space in our task, we turned on automatic relevance determination (ARD) while training the model. ARD allows the model to identify the directions in the input space that exhibit high relevance so as to select different length-scales for different directions.

\section{EXPERIMENT AND DATA COLlECTION}

In order to demonstrate the system's capability of learning from demonstrations in triadic interaction, experimental data were collected from the operation of a single subject working with a human assistant. The human assistant (i.e. the demonstrator) was considered to be an expert in driving the wheelchair remotely using the haptic interface. On the other hand, the subject, despite having experience in driving the wheelchair, had no prior acquaintance with the haptic joystick. During the experiment, the demonstrator monitored the operation of the wheelchair by observing its motion through rviz 3D visualization tool for ROS. She was provided with a frontal camera view of the environment, as well as laser scan data readings matching the map of the environment. The subject was asked to perform 12 laps (i.e. trials) in a cluttered lab area, where each lap involved visiting three checkpoints in sequence as shown in Fig. 3. Each lap was around 20 meters and on average took 45 seconds to complete.

During the trials, the demonstrator presented assistance whenever she saw fit, e.g. to steer the wheelchair from obstacles or to increase the pace of the task. The first trial was considered to be a practice trial, which aims at familiarizing

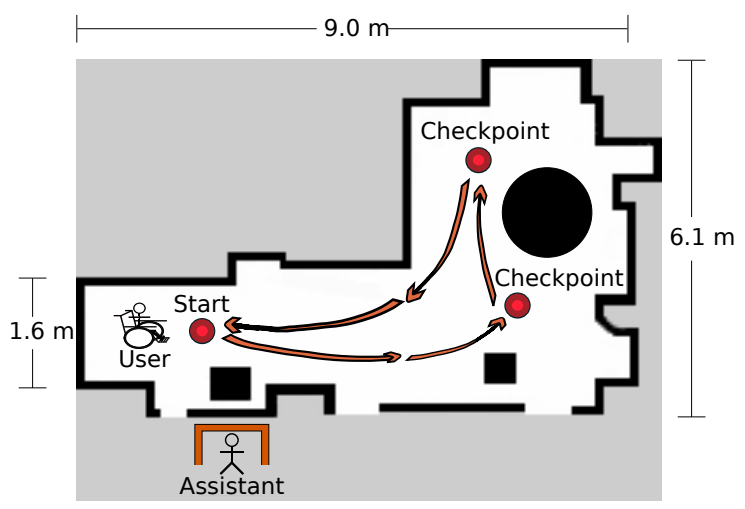

Fig. 3: The experimental task: A single trial involves going in sequence in between three checkpoints in the lab space. Black areas on the map denote obstacles. The assistant sits at one corner of the lab, monitors the operation of the user through a computer screen, and offers assistance. 

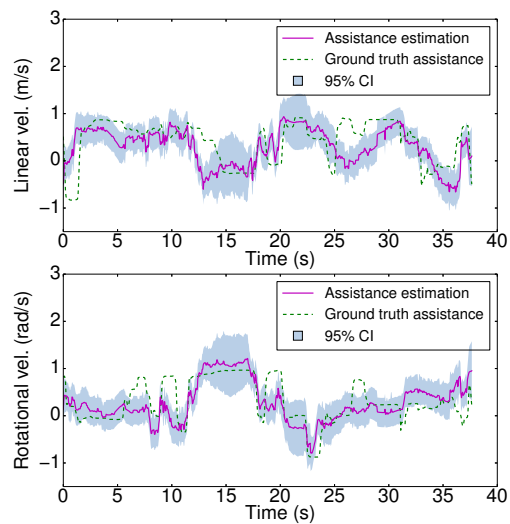
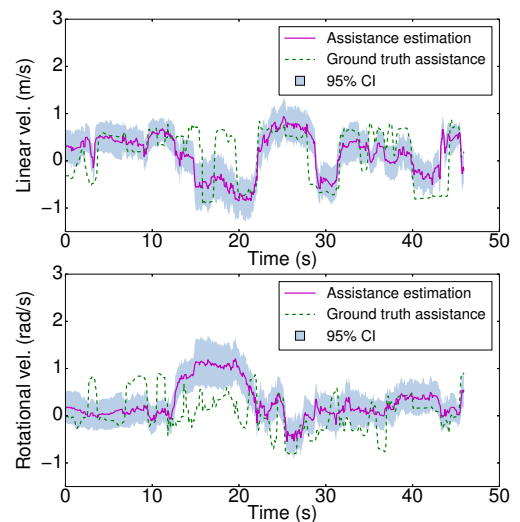
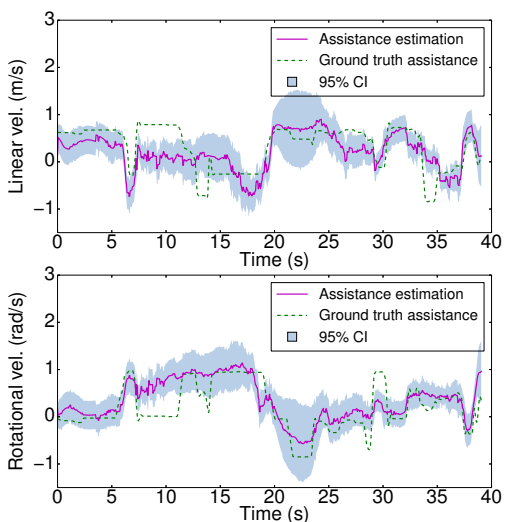

Fig. 4: Assistance command estimations for three representative trials. The model successfully estimates the assistance, following the trends in the ground truth data for assistant's commands.

the user with the triadic operation. Hence, the data collected during this trial were discarded from further analysis. The second trial was used as the one-shot demonstration session, the data from which were used to train the regression model. Remaining 10 trials were used as estimation trials, which allowed assessing the accuracy of the proposed model.

Sensor data were collected at $20 \mathrm{~Hz}$ to record the following environmental and user-related observations:

- Joint velocity command that moves ARTY,

- Laser scan readings in 15 directions,

- Minimum distance to an obstacle calculated using the laser scan readings,

- Velocity commands sent to ARTY by the user

to estimate target assistance commands:

- Velocity commands sent to ARTY by the assistant.

Note that the model learns the dynamics of the assistance behavior without any prior knowledge of the environment. Since the input variables involve only environmentindependent information, there is no need to build initial knowledge about the task space, such as building a map of the environment prior to the learning process.

\section{RESUlts AND Discussion}

Using the observations collected during the initial demonstration trial, we train a GP model for regression. This model is then used to estimate the assistance signals offered by the human in response to varying user commands and environmental context in the upcoming trials.

Figure 4 illustrates estimation profiles acquired using the proposed model for three representative trials. Evidently, model-based estimations follow trends similar to ground truth human assistance in both linear and rotational velocity commands, hence is able to mimic the operation of the human assistant.

Figure 5 presents the root mean squared error (RMSE) values between the assistance estimations and the actual assistance given by the human over all estimation trials for linear and rotational velocity commands. RMSE scores

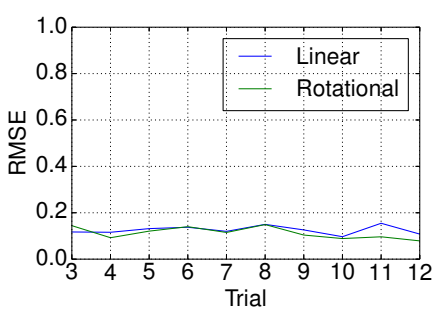

Fig. 5: RMSE scores showing the normalized estimation error over all trials.

tend to stay low, illustrating good and consistent estimation performance over the course of the experiment.

Figure 6 plots the paths followed by the wheelchair through all trials. Overlaying density plots mark the locations on the paths, where assistance was offered by the human demonstrator or was estimated. Figure 6 a plots the assistance density for the initial demonstration session. Figures $6 \mathrm{~b}$ and $6 \mathrm{c}$ plot the paths over the estimation trials (i.e. trials 3 to 12), and respectively present the ground truth assistance densities as demonstrated by the human and the assistance densities of the estimations. Upon closer inspection, it can be seen that the paths traversed by the wheelchair and the assistance offered by the human differs drastically from trial to trial. Additionally, the nature of the assistance does not strictly depend on the absolute location of the wheelchair on the map. For instance, even though strong assistance was offered in the middle of the path around coordinate (1.5, 0.5) during demonstration, some trials do not involve any such intervention in that region (e.g. trials 3,5 or 12). The GP model successfully captures this varying nature of the assistance so that the robotic wheelchair can learn to help the user in a similar fashion to the human assistant.

\section{CONCLUSiOnS AND FUTURE WORK}

This work is a first step in programming a robot that learns in one shot how and when to help a wheelchair user by observing assistance demonstrations offered by a human. We 

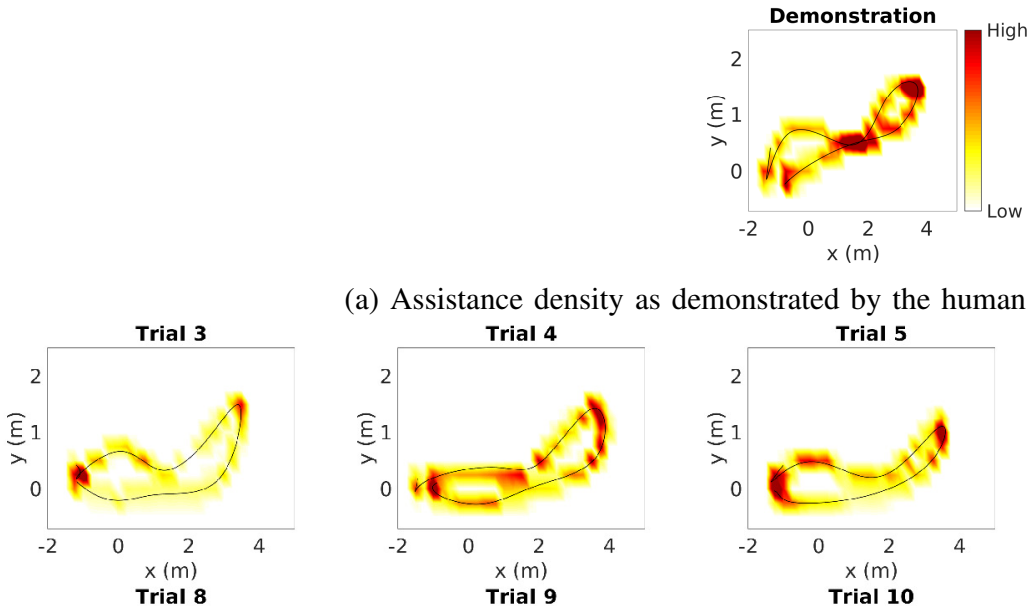

(a) Assistance density as demonstrated by the human in the demonstration trial
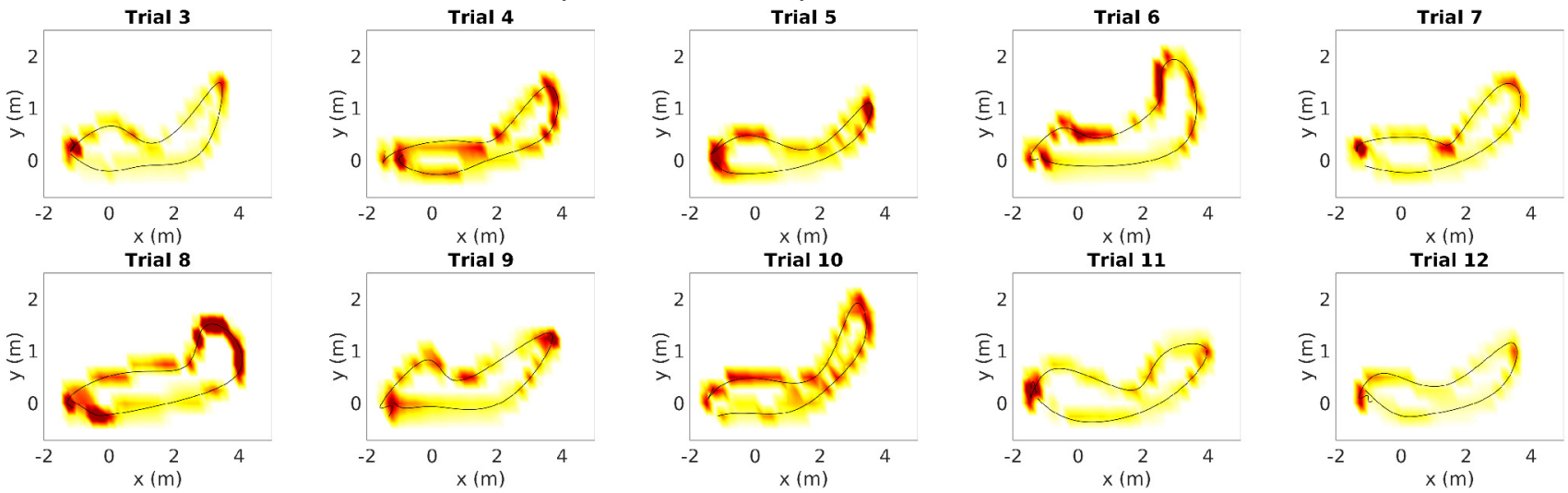

(b) Ground truth assistance densities as demonstrated by the human in the estimation trials
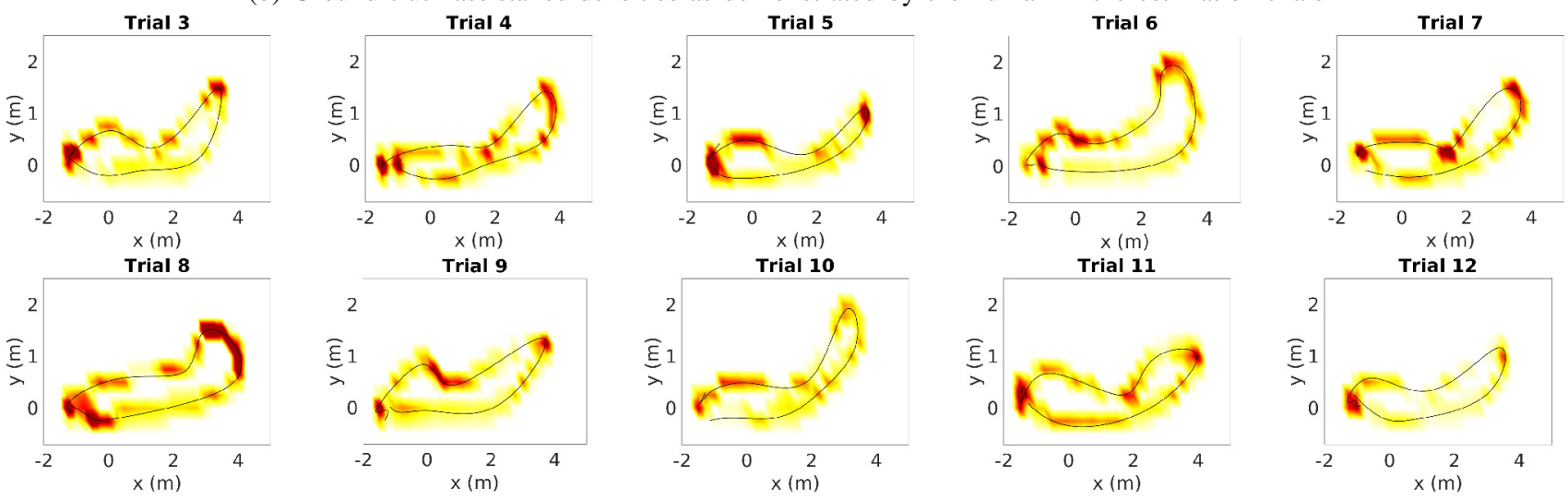

(c) Estimated assistance densities

Fig. 6: The paths taken through the trials with overlaying density plots that illustrate the whereabouts of the assistance offered by the human assistant. Darker red areas indicate higher amounts of assistance. a) Assistance density in the demonstration trial. This data were used to train the GP regression model. b) Ground truth assistance densities as offered by the human assistant. c) Estimated assistance densities.

train a GP regression model to learn the distribution that best describes the assistance function used by the human assistant. The model is able to learn the target function in one-shot, using data collected in a single demonstration session. A close inspection on estimation commands shows some low amplitude noise in signals in comparison to the ground truth assistance data. However, this noise might not bear any practical importance since many powered wheelchairs already come with "tremor damping", frequently with programmable joystick response sensitivity [30]. These signals can also be filtered in real time for smoother operation if they prove to be detectable by the users.

It is important to note that our approach is suitable for online assistance prediction. Even though training a GP model is computationally very costly (with complexity $\mathcal{O}\left(N^{3}\right)$, where $N$ is the size of the training set), the ability to learn in one-shot allows us to learn a model without considerable overhead due to the use of few training instances. Furthermore, the model created after the first trial can be considered as an initial model, and it is possible to train a new model on a parallel thread without pausing the operation whenever new assistance demonstrations are available. Within the scope of this paper, we have only demonstrated the estimation accuracy of the model. We are currently in the progress of implementing a predictive assistive mechanism for triadic interaction, where the wheelchair will autonomously respond to user's commands during ongoing action. An immediate future work will demonstrate the use of the proposed technique for online assistance prediction.

Our results indicate not only that the model can be used to 
estimate assistance signals that resemble human demonstrations, but also that the proposed technique can successfully generalize to different situations. The assistance can be estimated with high accuracy even when human assistance is presented in radically different moments. However, due to task selection, the generalization capability of the model can not be demonstrated fully. As a next step, we are going to investigate the model's ability to generalize to different situations by testing it in a trajectory that is completely different than that used in training.

This study reveals that the decisions of a human when offering assistance can be captured through the observation of only the user's behavior and the environmental context. However, this decision process is also strongly influenced by the characters and emotional states of the individuals participating in the task as well as their levels of task knowledge and expertise. For instance, the assistant in this experiment was inclined to offer a high level of assistance throughout the trials. However, it is possible that a different assistant would have gone for less intervention, in which case the system would have learned to be passive more often. Hence, any assistive system needs to be trained to accommodate such personal characteristics and should exploit individual decision processes of the actors. Our method presents a personalized user-modeling solution. Its capability to provide personalized assistance will be investigated in a follow-up study, where we will compare between policies learned for different user-assistant pairings.

\section{REFERENCES}

[1] The European Commission, "Commission staff working document on an action plan for the EU health workforce," 2012.

[2] A. Tapus and M. J. Mataric, "Emulating empathy in socially assistive robotics." in AAAI Spring Symposium: Multidisciplinary Collaboration for Socially Assistive Robotics, 2007, pp. 93-96.

[3] G. Colombo, M. Joerg, R. Schreier, V. Dietz, et al., "Treadmill training of paraplegic patients using a robotic orthosis," Journal of rehabilitation research and development, vol. 37, no. 6, pp. 693-700, 2000.

[4] L. L. Cai, A. J. Fong, C. K. Otoshi, Y. Liang, J. W. Burdick, R. R. Roy, and V. R. Edgerton, "Implications of assist-as-needed robotic step training after a complete spinal cord injury on intrinsic strategies of motor learning," The Journal of neuroscience, vol. 26, no. 41, pp. 10 564-10 568, 2006.

[5] R. Riener, L. Lunenburger, S. Jezernik, M. Anderschitz, G. Colombo, and V. Dietz, "Patient-cooperative strategies for robot-aided treadmill training: first experimental results," Neural Systems and Rehabilitation Engineering, IEEE Transactions on, vol. 13, no. 3, pp. 380-394, 2005.

[6] L. Kahn, W. Rymer, and D. Reinkensmeyer, "Adaptive assistance for guided force training in chronic stroke," in Engineering in Medicine and Biology Society, 2004. IEMBS'04. 26th Annual International Conference of the IEEE, vol. 1. IEEE, 2004, pp. 2722-2725.

[7] J. Hidler, D. Nichols, M. Pelliccio, K. Brady, D. D. Campbell, J. H. Kahn, and T. G. Hornby, "Multicenter randomized clinical trial evaluating the effectiveness of the lokomat in subacute stroke," Neurorehabilitation and Neural Repair, vol. 23, no. 1, pp. 5-13, 2009.

[8] E. C. Field-Fote and K. E. Roach, "Influence of a locomotor training approach on walking speed and distance in people with chronic spinal cord injury: a randomized clinical trial," Physical therapy, vol. 91, no. 1, pp. 48-60, 2011.

[9] H. Soh and Y. Demiris, "When and how to help: An iterative probabilistic model for learning assistance by demonstration," in IEEE/RSJ International Conference on Intelligent Robots and Systems (IROS), 2013, Nov 2013, pp. 3230-3236.
[10] C. Madan, A. Kucukyilmaz, M. Sezgin, and C. Basdogan, "Recognition of haptic interaction patterns in dyadic joint object manipulation," Haptics, IEEE Transactions on, vol. PP, no. 99, pp. 1-1, 2014.

[11] K. Reed and M. Peshkin, "Physical collaboration of human-human and human-robot teams," IEEE Transactions on Haptics, vol. 1, no. 2, pp. 108-120, July 2008.

[12] N. Stefanov, A. Peer, and M. Buss, "Role determination in humanhuman interaction," in WHC'09: IEEE World Haptics Conference, Salt Lake City, USA, 2009, pp. 51-56.

[13] R. Groten, D. Feth, R. Klatzky, and A. Peer, "The role of haptic feedback for the integration of intentions in shared task execution," IEEE Transactions on Haptics, vol. 6, no. 1, pp. 94-105, 2013.

[14] S. Calinon, F. Guenter, and A. Billard, "On learning, representing and generalizing a task in a humanoid robot," IEEE Transactions on Systems, Man and Cybernetics, Part B. Special issue on robot learning by observation, demonstration and imitation, vol. 37 , no. 2, pp. 286298, 2007.

[15] A. Billard, S. Calinon, R. Dillmann, and S. Schaal, "Robot programming by demonstration," in Springer Handbook of Robotics, B. Siciliano and O. Khatib, Eds. Springer, 2008, pp. 1371-1394.

[16] B. D. Argall, S. Chernova, M. Veloso, and B. Browning, "A survey of robot learning from demonstration," Robot. Auton. Syst., vol. 57, no. 5, pp. 469-483, May 2009.

[17] R. Gelin, J. Detriche, J. P. Lambert, and P. Malblanc, "The sprint of coach," in Systems, Man and Cybernetics, 1993. 'Systems Engineering in the Service of Humans', Conference Proceedings., International Conference on, vol. 3, 1993, pp. 547-552.

[18] T. Gomi and A. Griffith, "Developing intelligent wheelchairs for the handicapped." in Assistive Technology and Artificial Intelligence, ser. LNCS, V. O. Mittal, H. A. Yanco, J. M. Aronis, and R. C. Simpson, Eds., vol. 1458. Springer, 1998, pp. 150-178.

[19] R. Brooks, "A robust layered control system for a mobile robot," IEEE Journal of Robotics and Automation, vol. 2, no. 1, pp. 14-23, 1986.

[20] S. P. Levine, D. A. Bell, L. A. Jaros, R. C. Simpson, Y. Koren, and J. Borenstein, "The navchair assistive wheelchair navigation system," IEEE Trans. on Rehabilitation Engineering, vol. 7, pp. 443-451, 1999.

[21] E. S. Boy, C. L. Teo, and E. Burdet, "Collaborative wheelchair assistant," in IEEE/RSJ International Conference on Intelligent Robots and System, 2002, pp. 1511-1516.

[22] T. Carlson and Y. Demiris, "Human-wheelchair collaboration through prediction of intention and adaptive assistance." in ICRA. IEEE, 2008, pp. 3926-3931.

[23] — "Collaborative control for a robotic wheelchair: Evaluation of performance, attention, and workload." IEEE Transactions on Systems, Man, and Cybernetics, Part B, vol. 42, no. 3, pp. 876-888, 2012.

[24] E. Demeester, A. Hüntemann, D. Vanhooydonck, G. Vanacker, A. Degeest, H. V. Brussel, and M. Nuttin, "Bayesian estimation of wheelchair driver intents: Modeling intents as geometric paths tracked by the driver," in IEEE/RSJ International Conference on Intelligent Robots and Systems, IROS 2006, October 2006, pp. 5775-5780.

[25] H. Soh and Y. Demiris, "Spatio-temporal learning with the online finite and infinite echo-state gaussian processes," Neural Networks and Learning Systems, IEEE Transactions on, vol. 26, no. 3, pp. 522-536, March 2015.

[26] — , "Towards early mobility independence: An intelligent paediatric wheelchair with case studies," in IEEE/RSJ IROS2012 Workshop on Navigation and Manipulation Assistance for Robotic Wheelchairs, October 2012.

[27] M. Sarabia and Y. Demiris, "A humanoid robot companion for wheelchair users," in Social Robotics, ser. Lecture Notes in Computer Science, G. Herrmann, M. Pearson, A. Lenz, P. Bremner, A. Spiers, and U. Leonards, Eds., vol. 8239. Springer International Publishing, 2013, pp. 432-441.

[28] A. Kucukyilmaz, T. M. Sezgin, and C. Basdogan, "Intention recognition for dynamic role exchange in haptic collaboration," IEEE Transactions on Haptics, vol. 6, no. 1, pp. 58-68, First 2013.

[29] C. E. Rasmussen and C. K. I. Williams, Gaussian Processes for Machine Learning, ser. Adaptative computation and machine learning series. The MIT Press, 2006.

[30] B. E. Dicianno, R. A. Cooper, and J. Coltellaro, "Joystick control for powered mobility: Current state of technology and future directions," Physical Medicine and Rehabilitation Clinics of North America, vol. 21, no. 1, pp. $79-86,2010$, quality of Life. 